TTP07-07

SFB/CPP-07-12

MPP-2007-35

DESY 07-041

hep-ph/0703283

\title{
Electroweak corrections to large transverse momentum production of $W$ bosons at the LHC
}

\author{
Johann H. Kühn ${ }^{a}$, A. Kulesza ${ }^{b}$, S. Pozzorini ${ }^{c}$, M. Schulze ${ }^{a}$ \\ ${ }^{a}$ Institut für Theoretische Teilchenphysik, Universität Karlsruhe, \\ D-76128 Karlsruhe, Germany \\ ${ }^{b}$ Deutsches Elektronen-Synchrotron DESY, Notkestrasse 85, \\ D-22607 Hamburg, Germany \\ ${ }^{c}$ Max-Planck-Institut für Physik, Föhringer Ring 6, \\ D-80805 Munich, Germany
}

\begin{abstract}
:
To match the precision of present and future measurements of $W$-boson production at hadron colliders, electroweak radiative corrections must be included in the theory predictions. In this paper we consider their effect on the transverse momentum $\left(p_{\mathrm{T}}\right)$ distribution of $W$ bosons, with emphasis on large $p_{\mathrm{T}}$. We evaluate the full electroweak $\mathcal{O}(\alpha)$ corrections to the process $p p \rightarrow W j$ including virtual and real photonic contributions. We also provide compact approximate expressions which are valid in the high-energy region, where the electroweak corrections are strongly enhanced by logarithms of $\hat{s} / M_{W}^{2}$. These expressions include quadratic and single logarithms at one loop as well as quartic and triple logarithms at two loops. Numerical results are presented for proton-proton collisions at $14 \mathrm{TeV}$. The corrections are negative and their size increases with $p_{\mathrm{T}}$. At the LHC, where transverse momenta of $2 \mathrm{TeV}$ or more can be reached, the one- and two-loop corrections amount up to $-40 \%$ and $+10 \%$, respectively.
\end{abstract}

March 2007 


\section{Introduction}

The study of gauge-boson production has been among the primary goals of hadron colliders, starting with the discovery of the $W$ and $Z$ bosons more than two decades ago [1]. The investigation of the production dynamics, strictly predicted by the electroweak theory, constitutes one of the important tests of the Standard Model. Differential distributions of gauge bosons, in rapidity as well as in transverse momentum $\left(p_{\mathrm{T}}\right)$, have always been the subject of theoretical and experimental studies.

At large $p_{\mathrm{T}}$ the final state of the leading-order (LO) process consists of an electroweak gauge boson plus one recoiling jet. The high center-of-mass energy at the Large Hadron Collider (LHC) in combination with the enormous luminosity will allow to explore parton-parton scattering up to energies of several TeV and correspondingly production of gauge bosons with transverse momenta up to $2 \mathrm{TeV}$ or even beyond. In this energy range the electroweak corrections from virtual weakboson exchange are strongly enhanced, with the dominant terms in $L$-loop approximation being leading logarithms (LL) of the form $\alpha^{L} \log ^{2 L}\left(\hat{s} / M_{W}^{2}\right)$, next-to-leading logarithms (NLL) of the form $\alpha^{L} \log ^{2 L-1}\left(\hat{s} / M_{W}^{2}\right)$, and so on. These corrections, also known as electroweak Sudakov logarithms, may well amount to several tens of percent [2, 3, 4, 5, 6, 7]. A recent survey of the literature on electroweak Sudakov logarithms can be found in Ref. [8]. Specifically, the electroweak corrections to the $p_{\mathrm{T}}$ distribution of photons and $Z$ bosons at hadron colliders were studied in Refs. [5, 6]. In Ref. [6], it was found that at transverse momenta of $\mathcal{O}(1 \mathrm{TeV})$ the dominant two-loop contributions to these reactions amount to several percent and must be included to match the precision of the LHC experiments.

In this paper we study the electroweak corrections to the hadronic production of $W$ bosons at large $p_{\mathrm{T}}$. In contrast to the case of $Z$ and $\gamma$ production, the contributions from virtual and real photons cannot be separated from the purely weak corrections and will thus be included in our analysis.

The partonic reactions $\bar{q} q^{\prime} \rightarrow W^{ \pm} g(\gamma), q^{\prime} g \rightarrow W^{ \pm} q(\gamma)$ and $\bar{q} g \rightarrow W^{ \pm} \bar{q}^{\prime}(\gamma)$ with $q=u, d, s, c, b$ are considered. All of them are, however, trivially related by CP-and crossing-symmetry relations. Quark-mass effects are neglected throughout, which allows to incorporate the effect of quark mixing through a simple redefinition of parton distribution functions (see Sect. 2). The calculation of the virtual corrections is described in Sect. 3. In this Section we also present compact analytic expressions for the high-energy behaviour of the corrections which include quadratic and linear logarithms at one loop as well as quartic and triple logarithms at two loops. The calculation of the real corrections is performed using the dipole subtraction formalism [9, 10, 11, as described in Sect. 4. After convolution with parton distribution functions, we obtain radiatively corrected predictions for $p_{\mathrm{T}}$ distributions of $W$ bosons at the LHC, presented in Sect. 5. Concerning perturbative QCD, our predictions are based on the lowest order. To obtain realistic absolute cross sections, higher-order QCD corrections [12] must be included. However, the relative rates for $W^{+}, W^{-}$ and $Z$ production are expected to be more stable against QCD effects. Therefore, 
in Sect. 5 we also study the impact of the electroweak corrections on these ratios.

\section{Lowest order and kinematics}

The $p_{\mathrm{T}}$ distribution of $W$ bosons in the reactions $h_{1} h_{2} \rightarrow W^{ \pm} j$ is given by

$$
\frac{\mathrm{d} \sigma^{h_{1} h_{2}}}{\mathrm{~d} p_{\mathrm{T}}}=\sum_{i, j, k} \int_{0}^{1} \mathrm{~d} x_{1} \int_{0}^{1} \mathrm{~d} x_{2} \theta\left(x_{1} x_{2}-\hat{\tau}_{\min }\right) f_{h_{1}, i}\left(x_{1}, \mu^{2}\right) f_{h_{2}, j}\left(x_{2}, \mu^{2}\right) \frac{\mathrm{d} \hat{\sigma}^{i j \rightarrow W^{ \pm} k}}{\mathrm{~d} p_{\mathrm{T}}},
$$

where $\hat{\tau}_{\min }=\left(p_{\mathrm{T}}+m_{\mathrm{T}}\right)^{2} / s, m_{\mathrm{T}}=\sqrt{p_{\mathrm{T}}^{2}+M_{W}^{2}}$ and $\sqrt{s}$ is the collider energy. The indices $i, j$ denote initial-state partons and $f_{h_{1}, i}\left(x, \mu^{2}\right), f_{h_{2}, j}\left(x, \mu^{2}\right)$ are the corresponding parton distribution functions. $\hat{\sigma}^{i j \rightarrow W^{ \pm} k}$ is the partonic cross section for the subprocess $i j \rightarrow W^{ \pm} k$ and the sum runs over all $i, j, k$ combinations corresponding to the subprocesses

$$
\begin{aligned}
& \bar{u}_{m} d_{n} \rightarrow W^{-} g, \quad d_{n} \bar{u}_{m} \rightarrow W^{-} g, \quad g d_{n} \rightarrow W^{-} u_{m}, \quad d_{n} g \rightarrow W^{-} u_{m} \\
& \bar{u}_{m} g \rightarrow W^{-} \bar{d}_{n}, \quad g \bar{u}_{m} \rightarrow W^{-} \bar{d}_{n}
\end{aligned}
$$

for $W^{-}$production, and similarly for $W^{+}$production. The dependence of the partonic cross sections on the flavour indices $m, n$ amounts to an overall factor $\left|V_{u_{m} d_{n}}\right|^{2}$. This factor can be easily absorbed in the parton distribution functions of down-type quarks by redefining

$$
f_{h, d_{m}} \rightarrow \sum_{n=1}^{3}\left|V_{u_{m} d_{n}}\right|^{2} f_{h, d_{n}}, \quad f_{h, \bar{d}_{m}} \rightarrow \sum_{n=1}^{3}\left|V_{u_{m} d_{n}}\right|^{2} f_{h, \bar{d}_{n}}
$$

The partonic cross sections can then be computed using a trivial CKM matrix $\delta_{m n}$. The Mandelstam variables for the subprocess $i j \rightarrow W^{ \pm} k$ are defined in the standard way

$$
\hat{s}=\left(p_{i}+p_{j}\right)^{2}, \quad \hat{t}=\left(p_{i}-p_{W}\right)^{2}, \quad \hat{u}=\left(p_{j}-p_{W}\right)^{2} .
$$

Momentum conservation implies $\hat{s}+\hat{t}+\hat{u}=M_{W}^{2}$, and the invariants are related to the collider energy $s$ and the transverse momentum $p_{\mathrm{T}}$ by $p_{\mathrm{T}}^{2}=\hat{t} \hat{u} / \hat{s}$ with $\hat{s}=x_{1} x_{2} s$.

The $p_{\mathrm{T}}$ distribution for the unpolarized partonic subprocess $i j \rightarrow W^{ \pm} k$ reads

$$
\frac{\mathrm{d} \hat{\sigma}^{i j \rightarrow W^{ \pm} k}}{\mathrm{~d} p_{\mathrm{T}}}=\frac{p_{\mathrm{T}}}{8 \pi N_{i j} \hat{s}|\hat{t}-\hat{u}|}\left[\bar{\sum}\left|\mathcal{M}^{i j \rightarrow W^{ \pm} k}\right|^{2}+(\hat{t} \leftrightarrow \hat{u})\right],
$$

where $\bar{\sum}=\frac{1}{4} \sum_{\text {pol }} \sum_{\text {col }}$ involves the sum over polarization and color as well as the factor $1 / 4$ for averaging over initial-state polarization. The factor $1 / N_{i j}$ accounts for the initial-state colour average.

The unpolarized squared matrix elements for all partonic processes relevant for $W^{+}$and $W^{-}$production are related by crossing- and CP-symmetry relations. Thus the explicit computation of the unpolarized squared matrix element needs to be 
performed only once, e.g. for $\bar{u} d \rightarrow W^{-} g$. For this reaction, to lowest order in $\alpha$ and $\alpha_{\mathrm{S}}$, we have

$$
\bar{\sum}\left|\mathcal{M}_{\mathrm{Born}}^{\bar{u} d \rightarrow W^{-} g}\right|^{2}=32 \pi^{2} \frac{\alpha}{s_{\mathrm{W}}^{2}} \alpha_{\mathrm{S}} \frac{\hat{t}^{2}+\hat{u}^{2}+2 M_{W}^{2} \hat{s}}{\hat{t} \hat{u}}
$$

where $s_{\mathrm{W}}=\sqrt{1-c_{\mathrm{W}}^{2}}$ denotes the sine of the weak mixing angle.

\section{Virtual corrections}

The one-loop diagrams were reduced to a minimal set of coupling structures, standard matrix elements and scalar integrals as in Ref. [6]. The electroweak coupling constants were renormalized in the $G_{\mu}$-scheme, where $\alpha=\sqrt{2} G_{\mu} M_{W}^{2} s_{\mathrm{W}}^{2} / \pi$ is expressed in terms of the Fermi constant $G_{\mu}$ and $s_{\mathrm{W}}^{2}=1-M_{W}^{2} / M_{Z}^{2}$. Soft and collinear singularities resulting from virtual photons were regularized and combined with corresponding singularities from real photons as described in Sect. 4. Complete analytic results for the one-loop corrections and their asymptotic behaviour will be provided in Ref. [13. The numerical evaluation and detailed cross checks were performed with two independent programs. For the scalar loop integrals we used the Fortran library [14] and the FF library [15].

In the following, we present compact analytic expressions for the one- and twoloop NLL contributions at high energy. As in the case of $Z$ and $\gamma$ production [6], the NLL terms are obtained from the Born result by multiplication with a global factor. For the process $\bar{u} d \rightarrow W^{-} g$ we have

$$
\bar{\sum}\left|\mathcal{M}^{\bar{u} d \rightarrow W^{-} g}\right|^{2}=\bar{\sum}\left|\mathcal{M}_{\text {Born }}^{\bar{u} d \rightarrow W^{-} g}\right|^{2}\left[1+\left(\frac{\alpha}{2 \pi}\right) A^{(1)}+\left(\frac{\alpha}{2 \pi}\right)^{2} A^{(2)}\right] .
$$

At one loop, the NLL part consists of double- and single-logarithmic terms and reads

$$
A^{(1)} \stackrel{\mathrm{NLL}}{=}-C_{q_{\mathrm{L}}}^{\mathrm{ew}}\left(\mathrm{L}_{\hat{s}}^{2}-3 \mathrm{~L}_{\hat{s}}\right)-\frac{C_{\mathrm{A}}}{2 s_{\mathrm{W}}^{2}}\left(\mathrm{~L}_{\hat{t}}^{2}+\mathrm{L}_{\hat{u}}^{2}-\mathrm{L}_{\hat{s}}^{2}\right) .
$$

Here $C_{q_{\mathrm{L}}}^{\text {ew }}=Y_{q_{\mathrm{L}}}^{2} /\left(4 c_{\mathrm{W}}^{2}\right)+C_{\mathrm{F}} / s_{\mathrm{w}}^{2}, C_{\mathrm{F}}=3 / 4, C_{\mathrm{A}}=2$, and $\mathrm{L}_{\hat{r}}=\log \left(|\hat{r}| / M_{W}^{2}\right)$. At two loops we obtain

$$
\begin{aligned}
A^{(2) \stackrel{\mathrm{NLL}}{=}} & \frac{1}{2}\left(C_{q_{\mathrm{L}}}^{\mathrm{ew}}+\frac{C_{\mathrm{A}}}{2 s_{\mathrm{W}}^{2}}\right)\left[C_{q_{\mathrm{L}}}^{\mathrm{ew}}\left(\mathrm{L}_{\hat{s}}^{4}-6 \mathrm{~L}_{\hat{s}}^{3}\right)+\frac{C_{\mathrm{A}}}{2 s_{\mathrm{W}}^{2}}\left(\mathrm{~L}_{\hat{t}}^{4}+\mathrm{L}_{\hat{u}}^{4}-\mathrm{L}_{\hat{s}}^{4}\right)\right] \\
& +\frac{1}{6}\left[\frac{b_{1}}{c_{\mathrm{W}}^{2}}\left(\frac{Y_{q_{\mathrm{L}}}}{2}\right)^{2}+\frac{b_{2}}{s_{\mathrm{W}}^{2}}\left(C_{\mathrm{F}}+\frac{C_{\mathrm{A}}}{2}\right)\right] \mathrm{L}_{\hat{s}}^{3},
\end{aligned}
$$

where $b_{1}=-41 /\left(6 c_{\mathrm{w}}^{2}\right)$ and $b_{2}=19 /\left(6 s_{\mathrm{w}}^{2}\right)$. The NLL results (8) -(9) include the full electroweak corrections in the $M_{Z}=M_{W}$ approximation. The photonic singularities are separated using the fictitious photon mass 1$] \lambda=M_{W}$. Eq. (9) has

\footnotetext{
${ }^{1}$ For a discussion of this prescription, as well as for details concerning the implementation of the angular-dependent part of the NLL terms, we refer to Ref. [6].
} 
been derived from the general results for leading- and next-to-leading electroweak two-loop logarithms in Ref. [3].

\section{Real radiation}

Soft and collinear singularities from real radiation are treated using the dipole subtraction formalism [9, 10, 11]. Within this framework infrared singularities of a squared amplitude for real radiation are subtracted by an auxiliary function that has the same singular behaviour. This ensures that the phase space integral of the difference is a finite quantity and the integration can be performed numerically. The analytical result for the integral of the auxiliary function over the subspace of a radiated particle is then added to the result for virtual corrections. The singularities of the virtual corrections cancel against those of the integrated subtraction part.

The algorithms for constructing the auxiliary subtraction function and its integrated counterpart have been developed both for the case of photon radiation off massless or massive fermions [9] and QCD radiation off massless [10] or massive partons [11]. The latter [10, 11] approach can be easily adopted to the case of photon radiation. It employs dimensional regularization to regularize soft and collinear singularities while the former approach [9] introduces small photon and fermion masses. We have performed independent calculations of the virtual and real corrections within the two regularization schemes and verified that the soft and collinear (apart from initial-state and final-state collinear) singularities cancel. We also checked that the numerical results obtained within the massive and massless regularization scheme are in agreement. In both approaches we use expressions for the emission off a massive fermion to describe the emission off a $W$ boson, since only soft singularities are present in this case and they depend only on the charge of the external particle and not on its spin.

We restrict our analysis to $W$-boson production at high $p_{\mathrm{T}}$ by cutting away events with low $p_{\mathrm{T}}(W)$. Additionally, in order to avoid soft-gluon singularities which can arise as a side-effect of hard-photon radiation, we introduce a cut on $p_{\mathrm{T}}$ (jet).

The remaining initial-state collinear singularities are absorbed into the definition of parton distribution functions (PDFs). We choose to perform calculations in the $\overline{\mathrm{MS}}$ factorization scheme with the scale $M_{W}^{2}$. The photon-induced processes are not included in our calculations since they are expected to be suppressed due to the size of the electromagnetic coupling 2 .

\footnotetext{
${ }^{2}$ In order to consistently include $\mathcal{O}(\alpha)$ corrections in a calculation of a hadronic cross section, PDFs that are used in the calculation need to take into account QED effects. Such PDF analysis has been performed in [16] and the $\mathcal{O}(\alpha)$ effects are known to be small, both concerning the change in the quark distribution functions (below $\mathcal{O}(1 \%)$ [17] ) and the size of the photon distribution function. These effects are below typical uncertainty of hadronic processes. Moreover, the currently available PDFs incorporating $\mathcal{O}(\alpha)$ corrections, MRST2004QED [16, include QCD effects at the NLO in $\alpha_{\mathrm{S}}$. Since our calculations are of the lowest order in QCD, and QED effects on PDFs are estimated to be small, we choose to use a LO QCD PDF set without QED corrections incorporated, rather than MRST2004QED.
} 
For the gluon-induced subprocesses, final-state collinear singularities are also present. These singularities from collinear photon-quark configurations are avoided by recombining photons and quarks in the collinear region. In practice, we define the separation variable

$$
R(q, \gamma)=\sqrt{\left(\eta_{q}-\eta_{\gamma}\right)^{2}+\left(\phi_{q}-\phi_{\gamma}\right)^{2}}
$$

where $\eta_{i}$ is the pseudo-rapidity and $\phi_{i}$ is the azimuthal angle of a particle $i$. If $R(q, \gamma)<R_{\text {sep }}$, then the photon and quark momenta are recombined into an effective momentum $p_{\mathrm{T}}($ jet $)=p_{\mathrm{T}}(q+\gamma)$ which is subjected to the aforementioned cut.

\section{Predictions for the hadronic $W$ production at high transverse momentum}

For the numerical evaluation of the corrections we use the following input parameters: $G_{\mu}=1.16637 \times 10^{-5} \mathrm{GeV}^{-2}, M_{W}=80.39 \mathrm{GeV}, M_{Z}=91.19 \mathrm{GeV}$, $M_{t}=171.4 \mathrm{GeV}, M_{H}=120 \mathrm{GeV}$. Light-fermion and $b$-quark masses are neglected. The hadronic cross sections are obtained using LO MRST2001 PDFs [18]. We choose $\mu^{2}=p_{\mathrm{T}}^{2}(W)$ as the factorization scale and, similarly, as the scale at which the running strong coupling constant is evaluated. We also adopt, in agreement with the value used in the PDF analysis, the value $\alpha_{\mathrm{S}}\left(M_{Z}^{2}\right)=0.13$ and use the one-loop running expression for $\alpha_{\mathrm{S}}\left(\mu^{2}\right)$. For the numerical values of elements in the CKM quark mixing matrix we refer to [19]. The statistical accuracy of the $W j$ cross section at LHC is estimated using the integrated luminosity $\mathcal{L}=300 \mathrm{fb}^{-1}$, and the branching ratio $\operatorname{BR}\left(W \rightarrow e \nu_{e}+\mu \nu_{\mu}\right)=2 / 9$.

We apply the cuts $p_{\mathrm{T}}(W)>100 \mathrm{GeV}$ and $p_{\mathrm{T}}($ jet $)>100 \mathrm{GeV}$. The value of the separation parameter below which the recombination procedure is applied is taken to be $R_{\text {sep }}=0.1$.

The transverse momentum distributions in LO approximation for $p p \rightarrow W^{+} j$ and $p p \rightarrow W^{-} j$ at $\sqrt{s}=14 \mathrm{TeV}$ are shown in Fig. 19. In Fig. 1 b we plot the full $\mathcal{O}(\alpha)$ electroweak (NLO), one-loop next-to-leading logarithmic (NLL) and next-to-nextto-leading order (NNLO) corrections for $W^{+}$production. The NNLO corrections are defined as the NLO plus the two-loop NLL contributions (9). As expected, the NLO corrections increase significantly with $p_{\mathrm{T}}$. They result in a negative contribution ranging from $-15 \%$ at $p_{\mathrm{T}}=500 \mathrm{GeV}$ to $-42 \%$ at $p_{\mathrm{T}}=2 \mathrm{TeV}$. The one-loop NLL approximation (8) is in good agreement (at the 1-2\% level) with the full NLO result. The difference between NLO and NNLO curves is significant. As can be seen from the plot the two-loop terms are positive and amount to $+3 \%$ at $p_{\mathrm{T}}=1 \mathrm{TeV}$ and $+9 \%$ at $p_{\mathrm{T}}=2 \mathrm{TeV}$. The behaviour of the relative corrections for $W^{-}$production (Fig. 1r) is qualitatively and quantitatively very similar.

To underline the relevance of the large electroweak corrections at the LHC, in Fig. 2a and Fig. 2b we present the relative NLO and NNLO corrections to the $W^{+}$ 


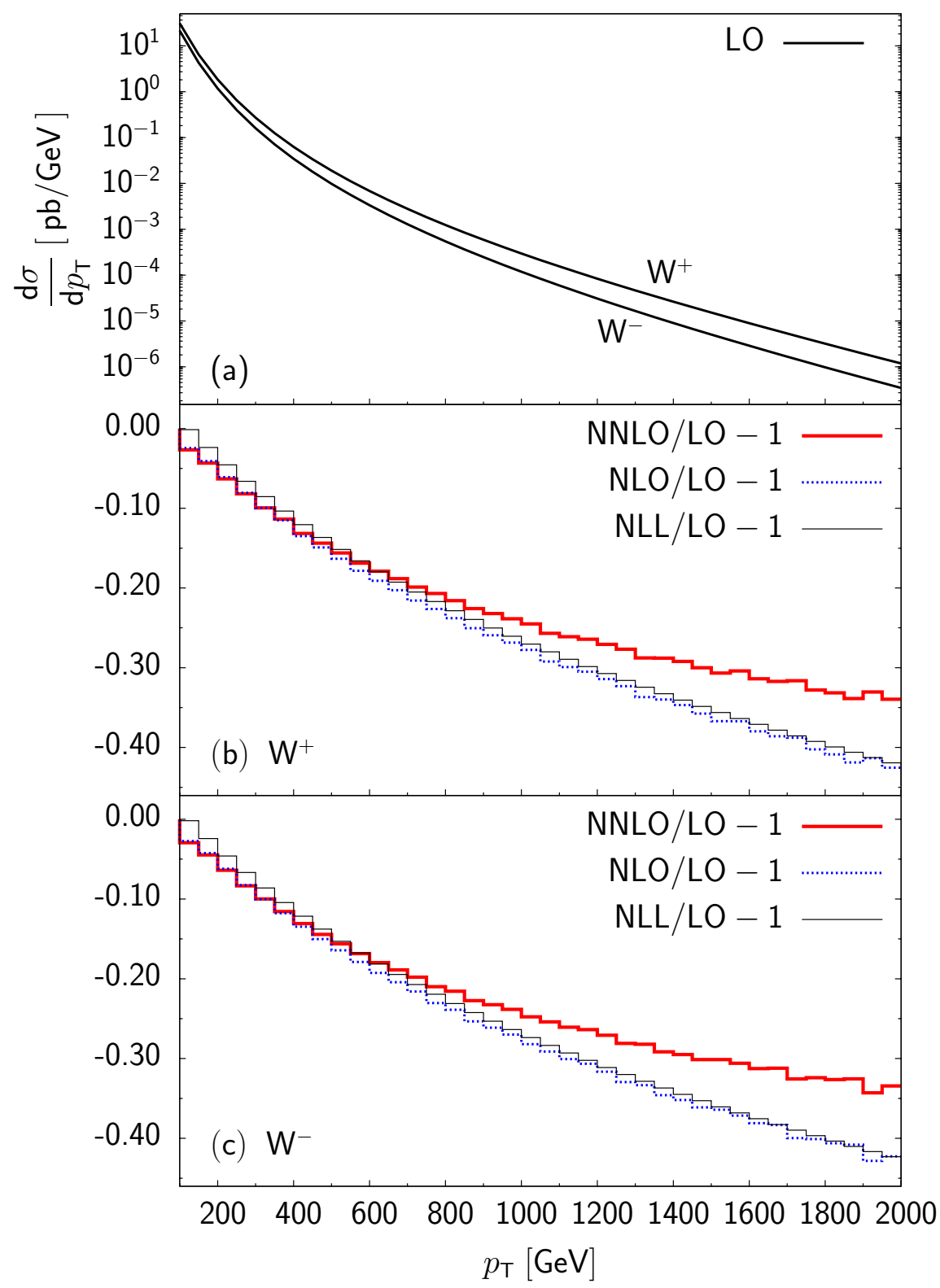

Figure 1: Transverse momentum distributions for $W$-boson production at the LHC: LO predictions (a) and relative electroweak corrections for $W^{+}$(b) and $W^{-}$(c) production in NLO (dotted), one-loop NLL (thin solid) and NNLO (thick solid) approximation. 


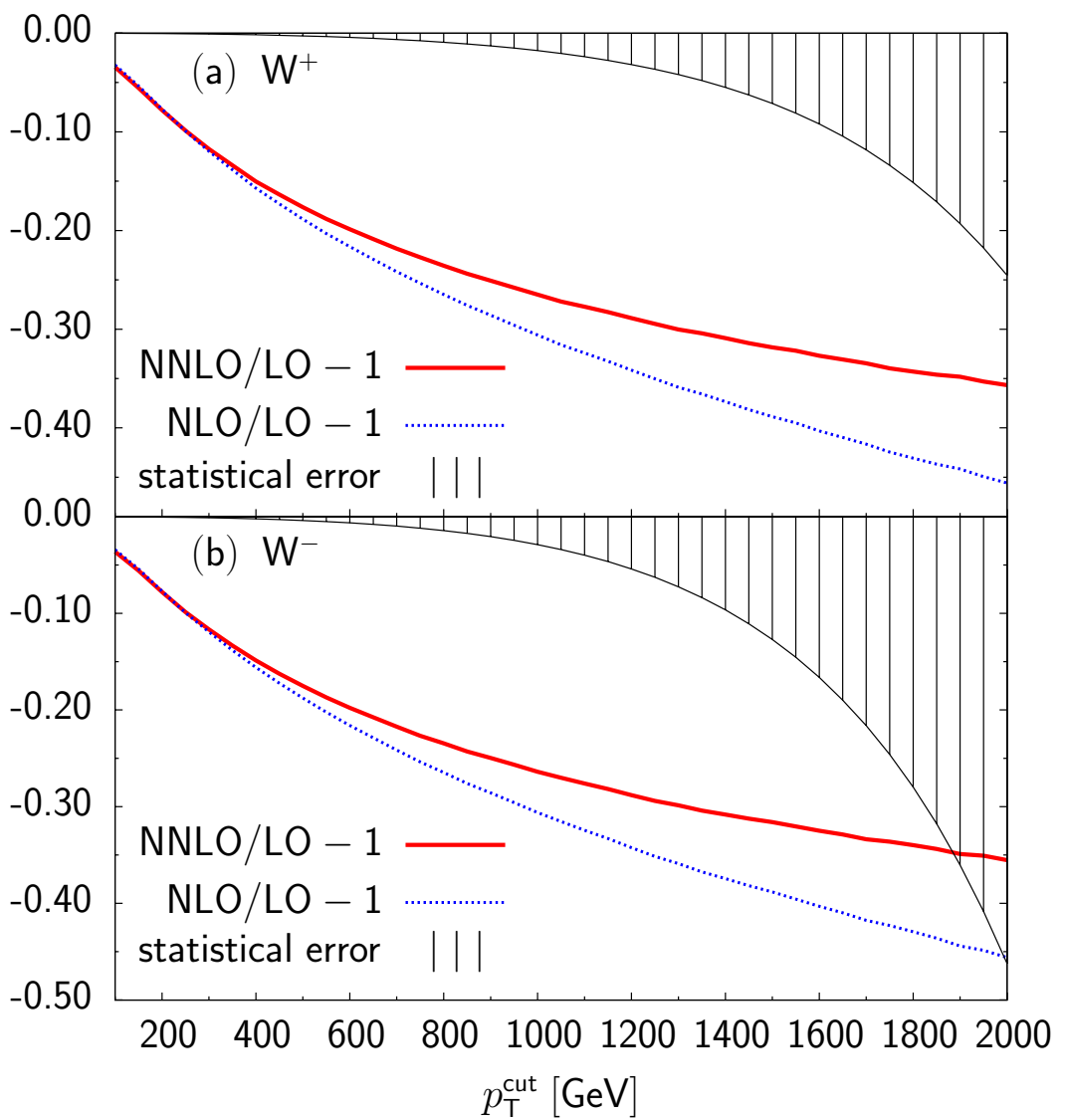

Figure 2: Unpolarized integrated cross section as a function of $p_{\mathrm{T}}^{\text {cut }}(W)$ for $W^{+}$ (a) and $W^{-}$(b) production: estimated statistical error (shaded area) and relative electroweak corrections in NLO (dotted) and NNLO (solid) approximation.

and $W^{-}$cross sections integrated over $p_{\mathrm{T}}$ starting from $p_{\mathrm{T}}=p_{\mathrm{T}}^{\text {cut }}$, as a function of $p_{\mathrm{T}}^{\text {cut }}$. This is compared with the statistical error, estimated as $\Delta \sigma_{\text {stat }} / \sigma=1 / \sqrt{N}$ with $N=\mathcal{L} \times \mathrm{BR} \times \sigma_{\mathrm{LO}}$ where the $\mathrm{BR}$ accounts for the $e \overline{\nu_{e}}$ and $\mu \overline{\nu_{\mu}}$ decay modes (for this estimate we ignore experimental efficiencies and cuts). It is clear that the size of the NLO corrections is much bigger then the statistical error. Also the difference between NNLO and NLO corrections, due to two-loop logarithmic effects, is significant. In terms of the estimated statistical error, these two-loop contributions amount to 1-3 standard deviations for $p_{\mathrm{T}}=\mathcal{O}(1 \mathrm{TeV})$.

Finally let us discuss the ratios of the $p_{\mathrm{T}}$ distributions for $W^{+}, W^{-}$and $Z$ bosons [6]. In contrast to the distributions themselves, these ratios are expected to be relatively insensitive to QCD corrections and theoretical uncertainties associated with $\alpha_{\mathrm{S}}$ and PDFs. Therefore they are good candidates for precision measurements. The $W^{+} / W^{-}$ratio is presented in Fig. [3a. In the considered $p_{\mathrm{T}}$ range the $\mathrm{LO}$ value increases from 1.5 to 3.5. As already observed, the (relative) electroweak corrections to the $W^{+}$and $W^{-}$production processes are almost identical. As a consequence 


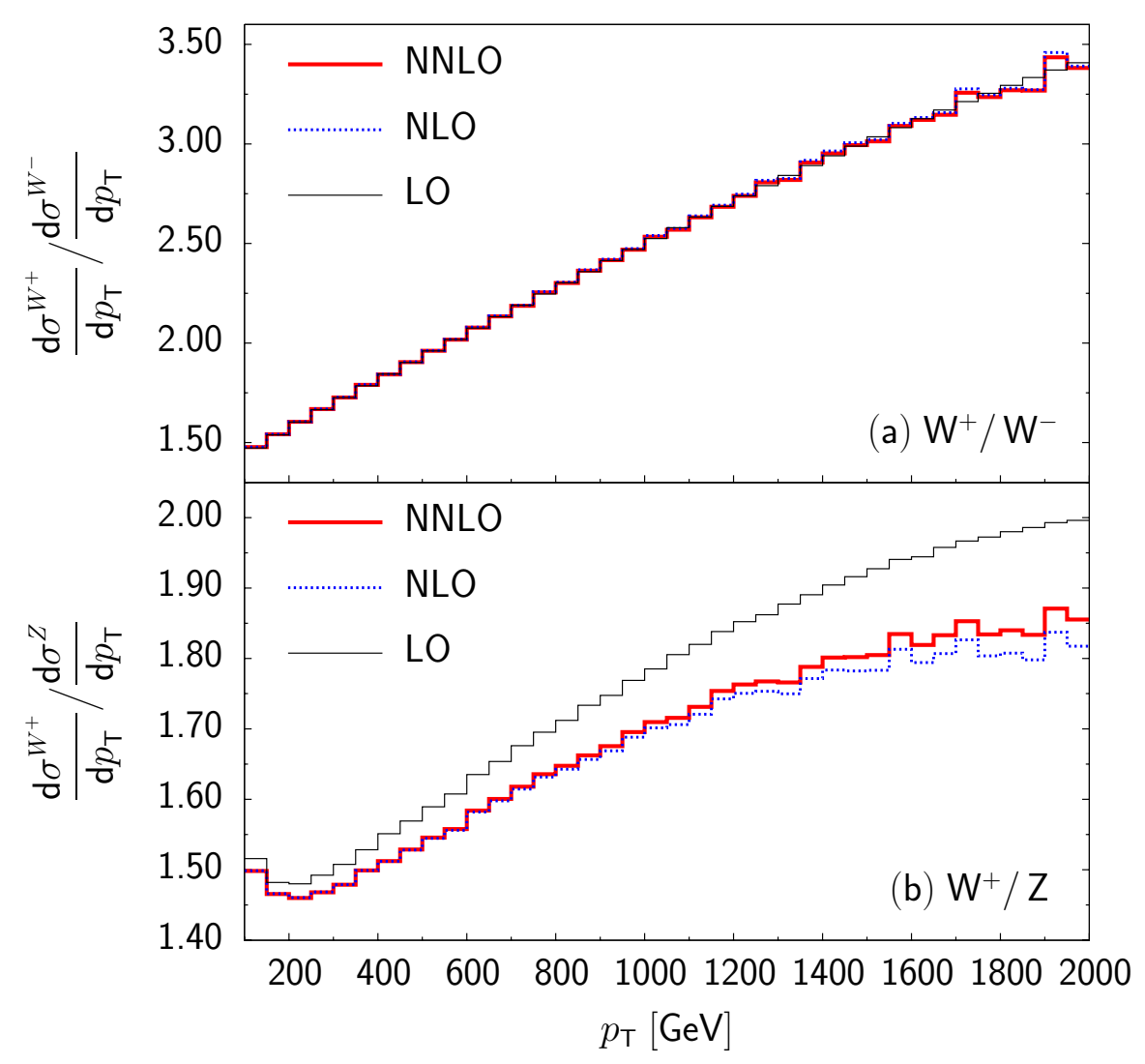

Figure 3: Ratio of the transverse momentum distributions for the processes (a) $p p \rightarrow W^{+} j$ and $p p \rightarrow W^{-} j$ and (b) $p p \rightarrow W^{+} j$ and $p p \rightarrow Z j$ at $\sqrt{s}=14 \mathrm{TeV}$ : LO (thin solid), NLO (dotted) and NNLO (thick solid) predictions.

the LO, NLO and NNLO curves in Fig. 3a overlap. In contrast, the impact of the electroweak corrections on the $W^{+} / Z$ ratio (Fig. 3 3 ) is clearly visible. Here the LO prediction, ranging from 1.5 to 2 , receives corrections that grow with $p_{\mathrm{T}}$ and amount to $5-10 \%$ for $p_{\mathrm{T}} \geq 1 \mathrm{TeV}$.

\section{Summary}

In this work we evaluated the electroweak corrections to large transverse momentum production of $W$ bosons at the LHC. The contributions from real and virtual photons cannot be separated in a gauge invariant manner from purely weak corrections and were thus included in our analysis. Soft and collinear singularities were regulated by introducing a small quark mass and a small photon mass and, alternatively, by using dimensional regularization. The real photon radiation was evaluated using the dipole formalism. The $\mathcal{O}(\alpha)$ corrections lead to a reduction of the cross section by about $15 \%$ at transverse momenta of $500 \mathrm{GeV}$ and reach more than $-40 \%$ at $2 \mathrm{TeV}$. 
We also derived a compact (NLL) approximation which includes the quadratic and linear logarithms and which gives a good description of the full $\mathcal{O}(\alpha)$ result with an accuracy of about 1-2\%. Considering the large event rate at the LHC, leading to a fairly good statistical precision even at transverse momenta up to $2 \mathrm{TeV}$, we evaluated also the dominant (NLL) two-loop terms. In the high- $p_{\mathrm{T}}$ region, these twoloop effects increase the cross section by $5-10 \%$ and thus become of importance in precision studies. We also studied the relative rates for $W^{+}, W^{-}$and $Z$ production, which are expected to be stable with respect to QCD effects. The electroweak corrections cancel almost completely in the $W^{+} / W^{-}$ratio. Instead, their impact on the $W^{+} / Z$ ratio is significant and amounts to several percent for $p_{\mathrm{T}} \geq 1 \mathrm{TeV}$.

\section{Acknowledgements}

We would like to thank S. Dittmaier, B. Jäger and P. Uwer for helpful discussions. This work was supported in part by BMBF Grant No. 05HT4VKA/3, the Sonderforschungsbereich Transregio 9 and the DFG Graduiertenkolleg "Hochenergiephysik und Teilchenastrophysik".

\section{References}

[1] G. Arnison et al. [UA1 Collaboration], Phys. Lett. B 122 (1983) 103; Phys. Lett. B 126 (1983) 398; M. Banner et al. [UA2 Collaboration], Phys. Lett. B 122 (1983) 476; P. Bagnaia et al. [UA2 Collaboration], Phys. Lett. B 129 (1983) 130.

[2] J. H. Kühn, A. A. Penin and V. A. Smirnov, Eur. Phys. J. C 17 (2000) 97 [hep-ph/9912503]; M. Ciafaloni, P. Ciafaloni and D. Comelli, Phys. Rev. Lett. D 84 (2000) 4810 [hep-ph/0001142]; A. Denner and S. Pozzorini, Eur. Phys. J. C 18 (2001) 461 [hep-ph/0010201]; Eur. Phys. J. C 21 (2001) 63 [hepph/0104127]; J. H. Kühn et al., Nucl. Phys. B 616 (2001) 286 [hep-ph/0106298]; B. Jantzen et al., Nucl. Phys. B 731 (2005) 188 [Erratum-ibid. B 752 (2006) 327] [hep-ph/0509157].

[3] V. S. Fadin et al., Phys. Rev. D 61 (2000) 094002 [hep-ph/9910338]; M. Melles, Phys. Rev. D 63 (2001) 034003 [hep-ph/0004056]; Phys. Rev. D 64 (2001) 054003 [hep-ph/0102097]; W. Beenakker and A. Werthenbach, Nucl. Phys. B 630 (2002) 3 [hep-ph/0112030]; A. Denner, M. Melles and S. Pozzorini, Nucl. Phys. B 662 (2003) 299 hep-ph/0301241]; S. Pozzorini, Nucl. Phys. B 692 (2004) 135 [hep-ph/0401087|.

[4] S. Dittmaier and M. Krämer, Phys. Rev. D 65 (2002) 073007 hep-ph/0109062];

E. Accomando, A. Denner and S. Pozzorini, Phys. Rev. D 65 (2002) 073003 [hep-ph/0110114]; W. Hollik and C. Meier, Phys. Lett. B 590 (2004) 69 
hep-ph/0402281; U. Baur and D. Wackeroth, Phys. Rev. D 70 (2004) 073015 |hep-ph/0405191|; E. Accomando, A. Denner and A. Kaiser, Nucl. Phys. B 706 (2005) 325 [hep-ph/0409247]; E. Accomando, A. Denner and C. Meier, Eur. Phys. J. C 47 (2006) 125 [hep-ph/0509234]; J. H. Kühn, A. Scharf and P. Uwer, Eur. Phys. J. C 45 (2006) 139 [hep-ph/0508092]; hep-ph/0610335; S. Moretti, M. R. Nolten and D. A. Ross, Phys. Lett. B 639 (2006) 513 [hep-ph/0603083]; C. M. Carloni Calame, G. Montagna, O. Nicrosini and A. Vicini, JHEP 0612 (2006) 016 [hep-ph/0609170]; W. Bernreuther, M. Fuecker and Z. G. Si, Phys. Rev. D 74 (2006) 113005 [hep-ph/0610334].

[5] E. Maina, S. Moretti and D. A. Ross, Phys. Lett. B 593 (2004) 143 [Erratumibid. B 614 (2005) 216] |hep-ph/0403050].

[6] J. H. Kühn, A. Kulesza, S. Pozzorini and M. Schulze, Phys. Lett. B 609 (2005) 277 [hep-ph/0408308]; Nucl. Phys. B 727 (2005) 368 hep-ph/0507178]; JHEP 0603 (2006) 059 [hep-ph/0508253].

[7] U. Baur, Phys. Rev. D 75 (2007) 013005 hep-ph/0611241.

[8] A. Denner, B. Jantzen and S. Pozzorini, Nucl. Phys. B 761 (2007) 1 [hep-ph/0608326].

[9] S. Dittmaier, Nucl. Phys. B 565 (2000) 69 hep-ph/9904440.

[10] S. Catani and M. H. Seymour, Nucl. Phys. B 485 (1997) 291 [Erratum-ibid. B 510 (1998) 503] hep-ph/9605323.

[11] S. Catani, S. Dittmaier, M. H. Seymour and Z. Trocsanyi, Nucl. Phys. B 627 (2002) 189 [hep-ph/0201036].

[12] R. K. Ellis, G. Martinelli and R. Petronzio, Nucl. Phys. B 211 (1983) 106; P. B. Arnold and M. H. Reno, Nucl. Phys. B 319 (1989) 37 [Erratum-ibid. B 330 (1990) 284]; R. J. Gonsalves, J. Pawlowski and C. F. Wai, Phys. Rev. D 40 (1989) 2245; W. T. Giele, E. W. N. Glover and D. A. Kosower, Nucl. Phys. B 403 (1993) 633 [hep-ph/9302225]; J. Campbell, R. K. Ellis and D. L. Rainwater, Phys. Rev. D 68 (2003) 094021 hep-ph/0308195|.

[13] J. H. Kühn, A. Kulesza, S. Pozzorini and M. Schulze, in preparation.

[14] A private library by A. Denner, based on W. Beenakker and A. Denner, Nucl. Phys. B 338, 349 (1990); A. Denner, U. Nierste and R. Scharf, Nucl. Phys. B 367 (1991) 637.

[15] G. J. van Oldenborgh, Comput. Phys. Commun. 66 (1991) 1.

[16] A. D. Martin, R. G. Roberts, W. J. Stirling and R. S. Thorne, Eur. Phys. J. C 39 (2005) 155 hep-ph/0411040. 
[17] M. Roth and S. Weinzierl, Phys. Lett. B 590 (2004) 190 hep-ph/0403200.

[18] A. D. Martin, R. G. Roberts, W. J. Stirling and R. S. Thorne, Phys. Lett. B 531 (2002) 216 hep-ph/0201127.

[19] S. Eidelman et al. [Particle Data Group], Phys. Lett. B 592 (2004) 1. 